\title{
Ausgewogen, durchdacht und vernünftig
}

\section{Marion Schafroth}

Dr. med., Vizepräsidentin EXIT Deutsche Schweiz

\author{
Die SAMW setzt in ihren Richtlinien auf Freiheit, Verantwortung und individuelle \\ Therapieentscheidungen mit der Arzt-Patienten-Beziehung als zentralem Element. \\ Dies sind Werte, die normalerweise auch durch die FMH hochgehalten werden. Die \\ Autorin hofft, dass die Ärztekammer sie auch dieses Mal verteidigt und im Standes- \\ recht festsetzt.
}

Der Senat der Schweizerischen Akademie der Medizinischen Wissenschaften (SAMW) hat am 17. Mai 2018 die neuen medizin-ethischen Richtlinien «Umgang mit Sterben und Tod» in Kraft gesetzt [1]. Diese betonen nicht nur die Wichtigkeit von Gesprächen über das Sterben und den Tod, sondern enthalten u.a. auch Empfehlungen in Bezug auf den Umgang mit Sterbewünschen, gemeinsame Entscheidungsfindung (Shared Decision Making), Vorausplanung von Behandlung und Betreuung (Advance Care Planning) und Einbezug der Angehörigen. Handlungen, die - möglicherweise oder sicher - den Eintritt des Todes beschleunigen, sind drei Kategorien zugeordnet:

- Allgemein akzeptierte Handlungen (Unterlassung und Abbruch lebenserhaltender Massnahmen, Linderung von Schmerzen und anderen Symptomen, Sedierung)

- Kontrovers diskutierte Handlungen (Suizidhilfe und Begleitung beim freiwilligen Verzicht auf Nahrung und Flüssigkeit)

- Verbotene ärztliche Handlungen (Tötung mit oder ohne Verlangen)

\section{Résumé}

Dans l'ensemble, les directives sont équilibrées, réfléchies et raisonnables. Elles sont désormais aussi fondamentalement conformes à l'ordre juridique suisse. Elles respectent I'autonomie du patient et du médecin. Elles intègrent en outre l'adaptation nécessaire de longue date à la pratique de I'assistance au suicide, qui a fait ses preuves depuis des années en Suisse [7]. Elles sont source de clarté pour le médecin et le guident dans la manière de gérer le désir d'assistance au suicide d'un patient avec sa propre conception de l'éthique. Que pourrait-on souhaiter de mieux?!
Wer die Richtlinien aufmerksam durchliest, wird feststellen, dass sie in ausgewogener Weise die beiden Pole "Patientenautonomie» und "Pflicht zur Fürsorge» berücksichtigen. Auch sind sie an die heute bereits bewährte medizinische Praxis und Rechtsprechung angepasst. So halten rechtskräftige Urteile des Verwaltungsgerichts Basel-Stadt vom 6. Juli 2017 sowie des Strafgerichts Basel vom 5. Juli 2012 fest, dass ärztliche Standesrichtlinien keine gesetzlichen Berufspflichten sind. Damit sind Ärzte berechtigt, ein Rezept für ein Sterbemedikament auch dann auszustellen, wenn die sterbewillige Person nicht unmittelbar vor dem natürlichen Ende ihres Lebens steht.

Die neuen Richtlinien werden von den Vorständen der FMH und der AGZ mit nicht stichhaltigen Argumenten bekämpft.

Dennoch werden die neuen Richtlinien nun von den Vorständen der FMH und der Ärztegesellschaft des Kantons Zürich AGZ mit nicht stichhaltigen Argumenten bekämpft [2-4]. Ihr Widerstand richtet sich vor allem gegen einen, aber wesentlichen Punkt: gegen das neu eingeführte Kriterium des «subjektiv unerträglichen Leidens» als Voraussetzung dafür, dass Ärztinnen und Ärzte Suizidhilfe leisten dürfen.

Es ist eine Frage von gesamtgesellschaftlicher Relevanz, zu der die Ärztekammer mit ihrem Entscheid im Oktober Stellung beziehen wird. Zwar handelt es sich bei der Suizidhilfe um ein Randphänomen, trotzdem wird darüber intensiv in Öffentlichkeit, Medien und Politik diskutiert. Die Mehrheit der Schweizer Bevölkerung will Zugang zur Suizidhilfe nicht nur am sich abzeichnendem Lebensende, sondern auch bei unerträglichem Leiden - und sie wünscht sich diese Hilfe von ärztlicher Seite [5]! Denn: Wer könnte die Nachvollzieh- 
barkeit eines Sterbewunsches besser beurteilen als die behandelnden Ärzte? Sie kennen ihre Patienten und ihr Krankengeschichte zum Teil seit Jahrzehnten und wissen am besten, ob mit einer neuen Therapie noch geholfen werden könnte oder ob alle palliativmedizinischen Massnahmen ausgeschöpft sind.

Der Vorstand der Ärztegesellschaft des Kantons Zürich macht in seiner Pressemitteilung hingegen keinen Hehl daraus, dass er den assistierten Suizid aufgrund von Leiden ohne unmittelbare Todesfolge ganz aus der Liste der ärztlichen Tätigkeiten streichen und an eine nicht-ärztliche (staatliche?) Stelle delegieren will, geregelt in der Patientenverfügung [4]. Diese nicht durchdachten Lösungsvorschläge sind aus vielen Gründen nicht umsetzbar und dürfen die Ärztekammer nicht dazu bewegen, die differenzierten Richtlinien der SAMW deshalb als Ganzes abzulehnen. Die Furcht vor der im Einzelfall manchmal schwierigen Auseinandersetzung zwischen den beiden Polen «Recht auf Freiheit, Menschenwürde und Privatsphäre» (bis hin zum Suizid) und «Recht auf Fürsorge» darf nicht dazu führen, dass die FMH die Suizidhilfe einfachheitshalber als dem ärztlichen Auftrag zuwi-

\section{Wer könnte die Nachvollziehbarkeit eines Sterbewunsches besser beurteilen als die behandelnden Ärzte?}

derlaufend ablehnt, wenn ein Mensch zwar subjektiv unerträglich leidet, aber das Lebensende noch fern scheint. Chronisches Leiden ist besonders schwer zu ertragen, wenn sich kein erlösendes Ende abzeichnet. Daher lässt sich Suizidhilfe auch beim nicht am Lebensende stehenden Menschen als eine ärztliche Handlung im Sinne einer Extremform von Leidenslinderung verstehen. Es ist ein Akt aus Respekt vor dem Leiden und eigenverantwortlichem Entscheid eines Mitmenschen - ein Akt von Humanität [6].

In den Richtlinien «Umgang mit Sterben und Tod» finden sich zum umstrittenen Punkt der Suizidhilfe u.a. auf den Seiten 24/25 folgende Erläuterungen:

"Insbesondere die Suizidhilfe wird kontrovers diskutiert. Angesichts des rechtlichen Rahmens für die Beihilfe zum Suizid in der Schweiz und der unterschiedlichen Haltungen im Umgang mit Sterbewünschen bei Ärztinnen, anderen medizinischen Fachpersonen und in der Öffentlichkeit lässt sich die Spannung zwischen den ethischen Forderungen nach Förderung der Selbstbestimmung und nach Schutz des Lebens nicht auflösen. Trotzdem sollen den medizinischen Fachpersonen möglichst klare Leitplanken gegeben werden ... Suizidhilfe ist keine medizinische Handlung, auf die Patienten einen Anspruch erheben könnten, sie ist jedoch eine rechtlich zulässige Tätigkeit. Sie kann vom Arzt geleistet werden, wenn er sich überzeugt hat, dass die untenstehenden Voraussetzungen erfüllt sind: ...»

\section{Diese Richtlinien begründen keine Pflicht zur Suizidhilfe.}

Diese Richtlinien begründen keine Pflicht zur Suizidhilfe. Ein Arzt muss nicht, aber er darf helfen - in Kooperation mit einer Sterbehilfeorganisation oder in alleiniger Verantwortung. Wenn er sich im Einzelfall dafür entscheidet, so bieten die Richtlinien taugliche Empfehlungen zum Vorgehen. Wenn er sich dagegen entscheidet, so wird er nicht unter Druck der Patienten geraten, weil Sterbehilfeorganisationen in diesem Fall wie bisher ihre Konsiliarärzte zuziehen.

\section{Fazit}

Die Richtlinien sind insgesamt ausgewogen, durchdacht und vernünftig. Neu sind sie nun auch grundsätzlich kongruent zur Schweizer Rechtsordnung. Sie achten die Autonomie von Patient und Arzt. Überdies erfüllen sie die längst notwendige Anpassung an die seit Jahren bewährte Praxis der Suizidhilfe in der Schweiz [7]. Für den einzelnen Arzt schaffen sie Klarheit und bieten Leitplanken, wie er dem Wunsch eines Patienten nach Suizidhilfe mit seinem eigenen ethischen Verständnis begegnen kann. Was kann man sich Besseres wünschen?!

\section{Literatur}

1 Kind C. Umgang mit Sterben und Tod. Schweiz Ärzteztg. 2018;99(24):791.

2 Widler J, Kohlbacher M. Suizid bei Krankheit. Schweiz Ärzteztg. 2018;99(30-31): 971-2.

3 Stellungnahme der FMH zu den SAMW-Richtlinien «Umgang mit Sterben und Tod» vom 22.2.2018: www.fmh.ch $\rightarrow$ Stellungnahmen

4 Medienmitteilung der AGZ vom 6.6.2018: https://www.aerzte-fuerzuerich.ch/medien/2018-06-06-lockerung-der-aerztlichen-suizidbeihilfe-bei-nicht-toedlichen-krankheiten-braucht

5 Umfrage Institut Link 20.4.2016: «Letzter Lebensabschnitt: Was erwartet die Bevölkerung vom Arzt?»: www.exit.ch $\rightarrow$ Buchhinweise

6 Schafroth M. Suizidhilfe - ein humanitärer Akt. Tages-Anzeiger, 16.6.2016.

7 Schafroth M. Suizidhilfe ist Teil der ärztlichen Aufgabe - die Zeit ist reif für eine Anpassung der SAMW-Richtlinien. Schweiz Ärzteztg. 2012;93(34)1226-7. 